\title{
Propagation of Rayleigh Wave in a Two-Temperature Generalized Thermoelastic Solid Half-Space
}

\author{
Baljeet Singh \\ Department of Mathematics, Post Graduate Government College, Sector 11, Chandigarh 160011, India \\ Correspondence should be addressed to Baljeet Singh; bsinghgcl1@gmail.com
}

Received 8 December 2012; Accepted 3 January 2013

Academic Editors: Y.-J. Chuo, D. Kass, and X. Perez-Campos

Copyright (C) 2013 Baljeet Singh. This is an open access article distributed under the Creative Commons Attribution License, which permits unrestricted use, distribution, and reproduction in any medium, provided the original work is properly cited.

\begin{abstract}
The Rayleigh surface wave is studied at a stress-free thermally insulated surface of an isotropic, linear, and homogeneous twotemperature thermoelastic solid half-space in the context of Lord and Shulman theory of generalized thermoelasticity. The governing equations of a two-temperature generalized thermoelastic medium are solved for surface wave solutions. The appropriate particular solutions are applied to the required boundary conditions to obtain the frequency equation of the Rayleigh wave. Some special cases are also derived. The speed of Rayleigh wave is computed numerically and shown graphically to show the dependence on the frequency and two-temperature parameter.
\end{abstract}

\section{Introduction}

Lord and Shulman [1] and Green and Lindsay [2] extended the classical dynamical coupled theory of thermoelasticity to generalized thermoelastic theories. These theories treat heat propagation as a wave phenomenon rather than a diffusion phenomenon and predict a finite speed of heat propagation. Ignaczak and Ostoja-Starzewski [3] explained in detail the above theories in their book Thermoelasticity with Finite Wave Speeds. The representative theories in the range of generalized thermoelasticity are reviewed by Hetnarski and Ignaczak [4]. Wave propagation in thermoelasticity has many applications in various engineering fields. Some problems on wave propagation in coupled or generalized thermoelasticity are studied by various researchers, for example, Deresiewicz [5], Sinha and Sinha [6], Sinha and Elsibai [7, 8], Sharma et al. [9], Othman and Song [10], Singh [11, 12], and many more.

Gurtin and Williams $[13,14]$ proposed the second law of thermodynamics for continuous bodies in which the entropy due to heat conduction was governed by one temperature, that of the heat supply by another temperature. Based on this law, Chen and Gurtin [15] and Chen et al. [16, 17] formulated a theory of thermoelasticity which depends on two distinct temperatures, the conductive temperature $\Phi$ and the thermodynamic temperature $T$. The two-temperature theory involves a material parameter $a^{*}>0$. The limit $a^{*} \rightarrow 0$ implies that $\Phi \rightarrow T$, and the classical theory can be recovered from two-temperature theory. The twotemperature model has been widely used to predict the electron and phonon temperature distributions in ultrashort laser processing of metals. According to Warren and Chen [18], these two temperatures can be equal in time-dependent problems under certain conditions, whereas $\Phi$ and $T$ are generally different in particular problems involving wave propagation. Following Boley and Tolins [19], they studied the wave propagation in the two-temperature theory of coupled thermoelasticity. They showed that the two temperatures, $T$ and $\Phi$, and the strain are represented in the form of a traveling wave plus a response, which occurs instantaneously throughout the body. Puri and Jordan [20] studied the propagation of harmonic plane waves in twotemperature theory. Quintanilla and Jordan [21] derived exact solutions of two initial-boundary value problems in the twotemperature theory with dual-phase-lag delay. Youssef [22] developed a theory of two-temperature generalized thermoelasticity. Kumar and Mukhopadhyay [23] extended the work of Puri and Jordan [20] in the context of the linear theory of two-temperature generalized thermoelasticity developed by Youssef [22]. Magaña and Quintanilla [24] showed the uniqueness and growth of solutions in two-temperature 
generalized thermoelastic theories. Recently, Youssef [25] formulated a theory of two-temperature thermoelasticity without energy dissipation.

In this paper, the Youssef [22] theory is followed for the theoretical study of the Rayleigh wave at the thermally insulated stress-free surface of an isotropic two-temperature thermoelastic solid half-space. The frequency equation of the Rayleigh wave is obtained. The frequency equation is also approximated by assuming small thermal coupling. The dependence of numerical values of the speed of the Rayleigh wave on material parameters, frequency, and twotemperature parameters is shown graphically for a particular material of the model.

\section{Basic Equations}

We consider an isotropic, linear, and homogeneous twotemperature thermoelastic solid half-space. Following Youssef [22], the governing equations for a two-temperature generalized thermoelastic half-space with one relaxation time are as follows:

(i) the stress-strain-temperature relations

$$
\sigma_{i j}=c_{i j k l} e_{k l}-\gamma_{i j}\left(T-\Phi_{0}\right),
$$

(ii) the displacement-strain relation

$$
e_{i j}=\frac{1}{2}\left(u_{i, j}+u_{j, i}\right) \text {, }
$$

(iii) the equation of motion

$$
\rho \ddot{u}_{i}=\sigma_{j i, j}+\rho F_{i},
$$

(iv) the energy equation

$$
-q_{i, i}=\rho T_{0} \dot{S}
$$

(v) the modified Fourier's law

$$
-K_{i j} \Phi_{, j}=q_{i}+\tau_{0} \dot{q}_{i}
$$

(vi) the entropy-strain-temperature relation

$$
\rho S=\frac{\rho c_{E}}{T_{0}} \theta+\gamma_{i j} e_{i j}
$$

Here, $\gamma_{i j}$ are the coupling parameters, $T$ is the mechanical temperature, $\Phi_{0}=T_{0}$ is the reference temperature, $\theta=T-T_{0}$ with $\left|\theta / T_{0}\right| \ll 1, \sigma_{i j}$ is the stress tensor, $e_{k l}$ is the strain tensor, $c_{i j k l}$ is the tensor of elastic constants, $\rho$ is the mass density, $q_{i}$ is the heat conduction vector, $K_{i j}$ is the thermal conductivity tensor, $c_{E}$ is the specific heat at constant strain, $u_{i}$ are the components of the displacement vector, $S$ is the entropy per unit mass, $\tau_{0}$ is the thermal relaxation time, which will ensure that the heat conduction equation will predict finite speeds of heat propagation, and $\Phi$ is the conductive temperature and satisfies the relation

$$
\Phi-T=a^{*} \Phi_{, i i},
$$

where $a^{*}>0$ is the two-temperature parameter.

\section{Analytical 2D Solution}

We consider a homogeneous and isotropic thermoelastic medium of an infinite extent with Cartesian coordinates system $(x, y, z)$, which is previously at uniform temperature. The origin is taken on the plane surface, and $z$-axis is taken normally into the medium $(z \geq 0)$. The surface $z=0$ is assumed to be stress-free and thermally insulated. The present study is restricted to the plane strain parallel to $x-z$ plane, with the displacement vector $\mathbf{u}=\left(u_{1}, 0, u_{3}\right)$. With the help of (1)-(3), we obtain the following two components of the equation of motion:

$$
\begin{aligned}
& (\lambda+2 \mu) u_{1,11}+(\lambda+\mu) u_{3,13}+\mu u_{1,33}-\gamma \theta_{, 1}=\rho \ddot{u}_{1}, \\
& (\lambda+2 \mu) u_{3,33}+(\lambda+\mu) u_{1,13}+\mu u_{3,11}-\gamma \theta_{, 3}=\rho \ddot{u}_{3} .
\end{aligned}
$$

Equations (4)-(6) lead to the following heat conduction equation:

$$
\begin{aligned}
K\left(\Phi_{, 11}+\Phi_{, 33}\right)= & \rho c_{E}\left(\dot{\theta}+\tau_{0} \ddot{\theta}\right) \\
& +\gamma T_{0}\left(\dot{u}_{1,1}+\tau_{0} \ddot{u}_{1,1}\right)+\gamma T_{0}\left(\dot{u}_{3,3}+\tau_{0} \ddot{u}_{3,3}\right)
\end{aligned}
$$

and (7) becomes

$$
\Phi-T=a^{*}\left(\Phi_{, 11}+\Phi_{, 33}\right) .
$$

The displacement components $u_{1}$ and $u_{3}$ are written in terms of potentials $q$ and $\psi$ as

$$
u_{1}=\frac{\partial q}{\partial x}-\frac{\partial \psi}{\partial z}, \quad u_{3}=\frac{\partial q}{\partial z}+\frac{\partial \psi}{\partial x}
$$

Using (11) in (8)-(10), we obtain

$$
\begin{gathered}
(\lambda+2 \mu)\left(\frac{\partial^{2} q}{\partial x^{2}}+\frac{\partial^{2} q}{\partial z^{2}}\right)-\gamma\left[\Phi-a^{*}\left(\frac{\partial^{2} \Phi}{\partial x^{2}}+\frac{\partial^{2} \Phi}{\partial z^{2}}\right)\right] \\
=\rho \frac{\partial^{2} q}{\partial t^{2}} \\
K\left(\Phi_{, 11}+\Phi_{, 33}\right) \\
=\rho c_{E}\left(\frac{\partial \Phi}{\partial t}+\tau_{0} \frac{\partial^{2} \Phi}{\partial t^{2}}\right) \\
\quad-a^{*} \rho c_{E}\left(1+\tau_{0} \frac{\partial}{\partial t}\right) \frac{\partial}{\partial t}\left(\frac{\partial^{2} \Phi}{\partial x^{2}}+\frac{\partial^{2} \Phi}{\partial z^{2}}\right) \\
+\gamma T_{0}\left(1+\tau_{0} \frac{\partial}{\partial t}\right) \frac{\partial}{\partial t}\left(\frac{\partial^{2} q}{\partial x^{2}}+\frac{\partial^{2} q}{\partial z^{2}}\right) \\
\mu\left(\frac{\partial^{2} \psi}{\partial x^{2}}+\frac{\partial^{2} \psi}{\partial z^{2}}\right)=\rho \frac{\partial^{2} \psi}{\partial t^{2}}
\end{gathered}
$$


For thermoelastic surface waves in the half-space propagating in $x$-direction, the potential functions $\Phi, q$, and $\psi$ are taken in the following form:

$$
(\Phi, q, \psi)=(\widehat{\Phi}(z), \widehat{q}(z), \widehat{\psi}(z)) \exp i(\eta x-\chi t),
$$

where $\chi^{2}=\eta^{2} c^{2}, \eta$ is the wave number, and $c$ is the phase velocity.

Substituting (15) with (12) and (13) and eliminating $\widehat{q}, \widehat{\Phi}$, we obtain the following auxiliary equation:

$$
D^{4}-A D^{2}+B=0
$$

where $D=d / d z$,

$$
\begin{gathered}
A=2 \eta^{2}+\eta^{2} c^{2}\left[\frac{c_{1}^{2}+\epsilon+\left(\bar{K}-a^{*} \chi^{2}\right)}{a^{*} \epsilon \chi^{2}-c_{1}^{2}\left(\bar{K}-a^{*} \chi^{2}\right)}\right], \\
B=\eta^{4}+\eta^{4} c^{2}\left[\frac{c_{1}^{2}+\epsilon+\left(\bar{K}-a^{*} \chi^{2}\right)}{a^{*} \epsilon \chi^{2}-c_{1}^{2}\left(\bar{K}-a^{*} \chi^{2}\right)}\right] \\
-\eta^{4} c^{4}\left[\frac{1}{a^{*} \epsilon \chi^{2}-c_{1}^{2}\left(\bar{K}-a^{*} \chi^{2}\right)}\right], \\
\epsilon=\frac{\bar{\gamma}^{2} T_{0}}{c_{E} \tau^{*}}, \quad \bar{K}=\frac{K}{\rho c_{E} \tau^{*}}, \quad \tau^{*}=\tau_{0}+\frac{i}{\chi}, \\
\bar{\gamma}=\frac{\gamma}{\rho}, \quad c_{1}^{2}=\frac{\lambda+2 \mu}{\rho} .
\end{gathered}
$$

With the help of (16) and keeping in mind that $\widehat{q}, \widehat{\Phi} \rightarrow 0$ as $z \rightarrow \infty$ for surface waves, the solutions $q$, $\Phi$ are written as

$$
\begin{gathered}
q=\left[A \exp \left(-\eta \beta_{1} z\right)+B \exp \left(-\eta \beta_{2} z\right)\right] \exp \iota(\eta x-\chi t), \\
\Phi=\left[\zeta_{1} A \exp \left(-\eta \beta_{1} z\right)+\zeta_{2} B \exp \left(-\eta \beta_{2} z\right)\right] \exp \iota(\eta x-\chi t),
\end{gathered}
$$

where

$$
\begin{gathered}
\beta_{1}{ }^{2}=\frac{1}{2 \eta^{2}}\left[A+\sqrt{A^{2}-4 B}\right], \\
\beta_{2}{ }^{2}=\frac{1}{2 \eta^{2}}\left[A-\sqrt{A^{2}-4 B}\right], \\
\zeta_{i}=\frac{\chi^{2}-c_{1}^{2}\left(1-\beta_{i}^{2}\right) \eta^{2}}{\bar{\gamma}\left[1+a^{*} \eta^{2}\left(1-\beta_{i}^{2}\right)\right]}, \quad(i=1,2) .
\end{gathered}
$$

Substituting (15) with (14) and keeping in mind that $\widehat{\psi} \rightarrow 0$ as $z \rightarrow \infty$ for surface waves, we obtain the following solution:

$$
\psi=C \exp \left[-\eta \beta_{3} z+\iota(\eta x-\chi t)\right]
$$

where

$$
\beta_{3}^{2}=1-\frac{c^{2}}{c_{2}^{2}}, \quad c_{2}^{2}=\frac{\mu}{\rho}
$$

\section{Derivation of Frequency Equation}

The mechanical and thermal conditions at the thermally insulated surface $z=0$ are as follows:

(i) vanishing of the normal stress component

$$
\sigma_{z z}=0,
$$

(ii) vanishing of the tangential stress component

$$
\sigma_{z x}=0 \text {, }
$$

(iii) vanishing of the normal heat flux component

$$
\frac{\partial \Phi}{\partial z}=0
$$

where

$$
\begin{aligned}
\sigma_{z z}= & \lambda\left(\frac{\partial^{2} q}{\partial x^{2}}+\frac{\partial^{2} q}{\partial z^{2}}\right)+2 \mu\left(\frac{\partial^{2} \psi}{\partial x \partial z}\right) \\
& +2 \mu \frac{\partial^{2} q}{\partial z^{2}}-\gamma\left[\Phi-a^{*}\left(\frac{\partial^{2} \Phi}{\partial x^{2}}+\frac{\partial^{2} \Phi}{\partial z^{2}}\right)\right], \\
\sigma_{z x} & =\mu\left[2 \frac{\partial^{2} q}{\partial x \partial z}-\frac{\partial^{2} \psi}{\partial z^{2}}+\frac{\partial^{2} \psi}{\partial x^{2}}\right] .
\end{aligned}
$$

Making use of solutions (18) and (21) for $q$, $\Phi$, and $\Psi$ in (23) to (25) and eliminating $A, B$, and $C$, we obtain the following equation:

$$
\begin{aligned}
4 \mu \eta^{2} \beta_{1} \beta_{2} \beta_{3}\left(\zeta_{2}-\zeta_{1}\right)-\left(1+\beta_{3}^{2}\right) & \\
\times & {\left[\eta^{2}\left\{-\lambda\left(\zeta_{2} \beta_{2}-\zeta_{1} \beta_{1}\right)+(\lambda+2 \mu) \beta_{1} \beta_{2}\left(\zeta_{2} \beta_{1}-\zeta_{1} \beta_{2}\right)\right\}\right.} \\
& \left.-\rho c_{1}^{2} \zeta_{1} \zeta_{2}\left(\beta_{2}-\beta_{1}\right)\left\{1+a^{*} \eta^{2}\left(1+\beta_{1} \beta_{2}\right)\right\}\right]=0,
\end{aligned}
$$

which is the frequency equation of thermoelastic Rayleigh wave in a two-temperature generalized thermoelastic medium in the context of the Lord and Shulman [1] theory. 


\section{Special Cases}

5.1. Small Thermal Coupling $(\epsilon \ll 1)$. For most of materials, $\epsilon$ is small at a normal temperature. For $\epsilon \ll 1$ and using (19), we obtain the following approximated relations as

$$
\begin{array}{rl}
\beta_{1}^{2}+ & \beta_{2}{ }^{2} \\
\equiv & 2-c^{2}\left\{\frac{c_{1}^{2}+\left(\bar{K}-a^{*} \chi^{2}\right)}{c_{1}{ }^{2}\left(K-a^{*} \chi^{2}\right)}\right\} \\
& \times\left[1+\epsilon\left\{\frac{1}{c_{1}{ }^{2}+\left(\bar{K}-a^{*} \chi^{2}\right)}+\frac{a^{*} \chi^{2}}{c_{1}{ }^{2}\left(\bar{K}-a^{*} \chi^{2}\right)}\right\}\right], \\
\beta_{1}{ }^{2}{ }_{2}^{2} & 1-c^{2}\left\{\frac{c_{1}{ }^{2}+\left(\bar{K}-a^{*} \chi^{2}\right)}{c_{1}{ }^{2}\left(\bar{K}-a^{*} \chi^{2}\right)}\right\} \\
\equiv & {\left[1+\epsilon\left\{\frac{1}{c_{1}{ }^{2}+\left(\bar{K}-a^{*} \chi^{2}\right)}+\frac{a^{*} \chi^{2}}{c_{1}{ }^{2}\left(\bar{K}-a^{*} \chi^{2}\right)}\right\}\right]} \\
& +\frac{c^{4}}{c_{1}{ }^{2}\left(\bar{K}-a^{*} \chi^{2}\right)}\left[1+\epsilon \frac{a^{*} \chi^{2}}{c_{1}{ }^{2}\left(\bar{K}-a^{*} \chi^{2}\right)}\right] .
\end{array}
$$

With the help of these approximations, we can approximate $\beta_{1}, \beta_{2}$, and the coupling coefficients $\zeta_{1}$ and $\zeta_{2}$. Finally, the frequency equation (27) can be approximated. Further, if we consider $\epsilon \rightarrow 0, a^{*} \rightarrow 0$, we obtain $\beta_{1} \equiv \sqrt{1-c^{2} / c_{1}^{2}}$ and $\beta_{2} \equiv \sqrt{1-c^{2} / \bar{K}}$

For numerical purpose, we put $c^{2}=c^{* 2}+\epsilon\left(\xi_{1}+i \xi_{2}\right)$, where $c^{*}$ is the classical Rayleigh wave velocity, and $\xi_{1}$ and $\xi_{2}$ are two reals, then

$$
\eta=\frac{\chi}{c^{*}}\left(1-\frac{\epsilon \xi_{1}}{2 c^{* 2}}-i \frac{\epsilon \xi_{2}}{2 c^{* 2}}\right)
$$

The velocity of propagation is equal to $\left(c^{*}+\epsilon \xi_{1} / 2 c^{*}\right)$, and the amplitude attenuation factor is equal to $\exp \left[\epsilon \chi \xi_{2} x / 2 c^{* 3}\right]$ with $\xi_{2}<0$.

5.2. Small Frequency $(\chi \ll 1)$. For $\chi \ll 1$ and using (19), we obtain the following approximated relations as

$$
\begin{aligned}
\beta_{1}^{2}+\beta_{2}{ }^{2} \equiv & 2-c^{2}\left\{\frac{c_{1}^{2}+\epsilon+\bar{K}}{c_{1}{ }^{2} \bar{K}}\right\} \\
& \times\left[1+\chi^{2}\left\{\frac{(1+\epsilon) a^{*}}{c_{1}{ }^{2} \bar{K}}-\frac{a^{*}}{c_{1}{ }^{2}+\epsilon+\bar{K}}\right\}\right],
\end{aligned}
$$

$$
\begin{aligned}
\beta_{1}^{2} \beta_{2}^{2} \equiv & 1-c^{2}\left\{\frac{c_{1}{ }^{2}+\epsilon+\bar{K}}{c_{1}{ }^{2} \bar{K}}\right\} \\
& \times\left[1+\chi^{2}\left\{\frac{(1+\epsilon) a^{*}}{c_{1}{ }^{2} \bar{K}}-\frac{a^{*}}{c_{1}^{2}+\epsilon+\bar{K}}\right\}\right] \\
& +\frac{c^{4}}{c_{1}{ }^{2} \bar{K}}\left[1+\chi^{2} \frac{(1+\epsilon) a^{*}}{c_{1}{ }^{2} \bar{K}}\right] .
\end{aligned}
$$

With the help of these approximations, we can approximate $\beta_{1}, \beta_{2}$, and the coupling coefficients $\zeta_{1}$ and $\zeta_{2}$. Finally, the frequency equation (27) can be approximated. Also, if we consider $\chi \rightarrow 0, \epsilon \rightarrow 0$, we obtain $\beta_{1} \equiv \sqrt{1-c^{2} / c_{1}^{2}}$ and $\beta_{2} \equiv \sqrt{1-c^{2} / \bar{K}}$

5.3. Isotropic Elastic Case. If we neglect thermal parameters, then the frequency equation (27) reduces to

$$
\left(2-\frac{c^{2}}{c_{2}^{2}}\right)^{2}=4 \sqrt{1-\frac{c^{2}}{c_{1}^{2}}} \sqrt{1-\frac{c^{2}}{c_{2}^{2}}},
$$

which is the frequency equation of Rayleigh wave for an isotropic elastic case.

\section{Numerical Example}

The speed of propagation is computed for the following material parameters of aluminium metal: $\lambda=5.775 \times$ $10^{11} \mathrm{Dyn} \cdot \mathrm{cm}^{-2}, \mu=2.646 \times 10^{11} \mathrm{Dyn} \cdot \mathrm{cm}^{-2}, \rho=2.7 \mathrm{~g} \cdot \mathrm{cm}^{-3}$, $c_{E}=0.236 \mathrm{Cal} \cdot \mathrm{g}^{-1} \cdot{ }^{\circ} \mathrm{C}^{-1}, K=0.492 \mathrm{Cal} \cdot \mathrm{cm}^{-1} \cdot \mathrm{s}^{-1} \cdot{ }^{\circ} \mathrm{C}^{-1}$, $\gamma=0.05(3 \lambda+2 \mu), T_{0}=27^{\circ} \mathrm{C}$, and $c^{*}=0.9554$.

The nondimensional speed of Rayleigh wave is shown graphically against the frequency $(2-20 \mathrm{~Hz})$ in Figure 1, when the two-temperature parameter $a^{*}=0.5$. With the increase in frequency, it increases very sharply to its maximum value near $\chi=4.3 \mathrm{~Hz}$, and then it decreases slowly for the higher frequency range. The nondimensional speed of Rayleigh wave is also shown graphically against the two-temperature parameter $a^{*}$ in Figure 2 for $\chi=10 \mathrm{~Hz}$. With the increase in the value of two-temperature parameter, it increases very sharply to its maximum value at $a^{*}=0.47$. Thereafter, it decreases sharply to its minimum value.

\section{Conclusion}

The appropriate solutions of all the governing equations of a two-temperature generalized thermoelastic medium are applied at the boundary conditions at a thermally insulated free surface of a half-space to obtain the frequency equation of Rayleigh wave in the context of the Lord and Shulman [1] theory. The frequency equation is approximated for the case of small thermal coupling and small frequency and reduced for isotropic elastic case. From the frequency equation of Rayleigh wave, it is observed that the phase speed of Rayleigh wave depends on various material parameters including the two-temperature parameter. The dependence of numerical 


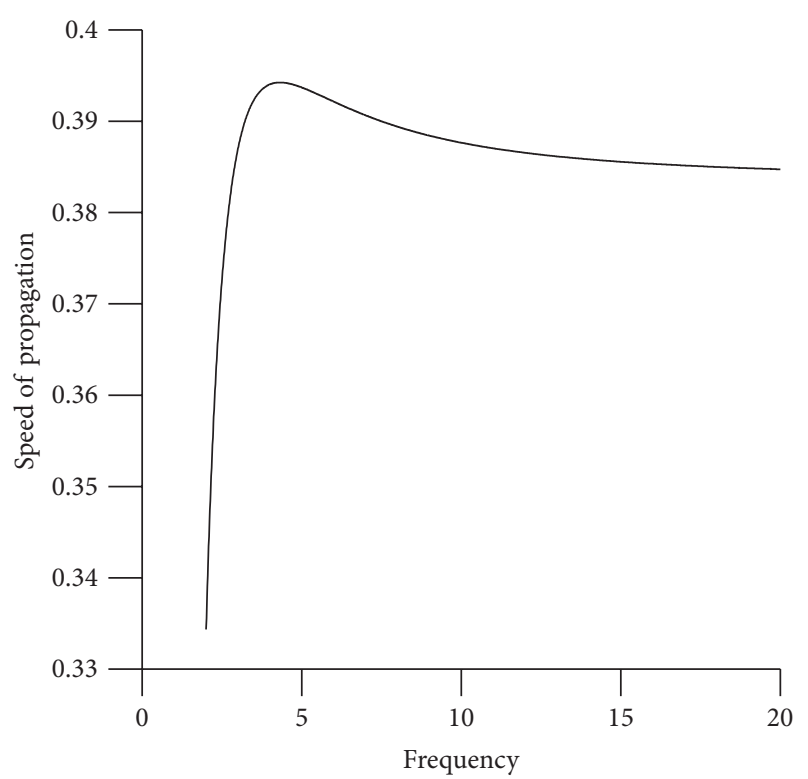

FIGURE 1: Variations of the nondimensional speed of propagation of Rayleigh wave against the frequency $(\chi)$ when $a^{*}=0.5$.

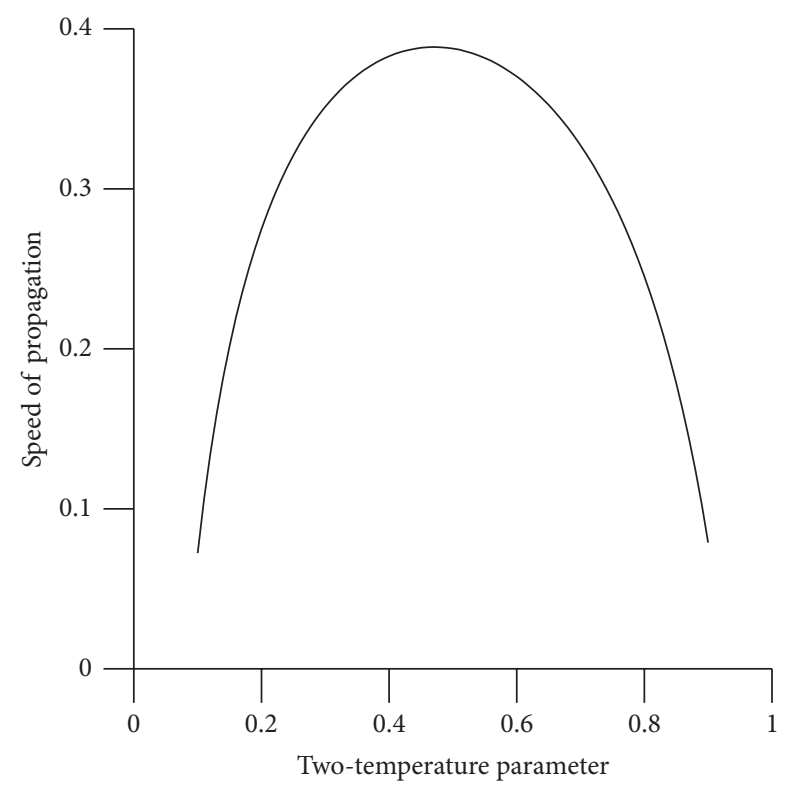

FIGURE 2: Variations of the nondimensional speed of propagation of Rayleigh wave against the two-temperature parameter $\left(a^{*}\right)$ when $\chi=10 \mathrm{~Hz}$.

values of speed of propagation on the frequency and twotemperature parameter is shown graphically for a particular material representing the model. The present problem is of geophysical interest, particularly in investigations concerned with earthquakes and other phenomena in seismology. The applications range from geophysical problems to quantitative nondestructive evaluation of mechanical structures and acoustic tomography for medical purposes. The problem though is theoretical, but it can provide useful information for experimental researchers working in the field of geophysics and earthquake engineering and seismologist working in the field of mining tremors and drilling into the crust of the earth.

\section{References}

[1] H. W. Lord and Y. Shulman, "A generalized dynamical theory of thermoelasticity," Journal of the Mechanics and Physics of Solids, vol. 15, no. 5, pp. 299-309, 1967.

[2] A. E. Green and K. A. Lindsay, "Thermoelasticity, Journal of Elasticity, vol. 2, no. 1, pp. 1-7, 1972.

[3] J. Ignaczak and M. Ostoja-Starzewski, Thermoelasticity with Finite Wave Speeds, Oxford University Press, Oxford, UK, 2009.

[4] R. B. Hetnarski and J. Ignaczak, "Generalized thermoelasticity," Journal of Thermal Stresses, vol. 22, no. 4, pp. 451-476, 1999.

[5] H. Deresiewicz, "Effect of boundaries on waves in a thermoelastic solid: reflexion of plane waves from a plane boundary," Journal of the Mechanics and Physics of Solids, vol. 8, no. 3, pp. 164-172, 1960.

[6] A. N. Sinha and S. B. Sinha, "Reflection of thermoelastic waves at a solid half-space with thermal relaxation," Journal of Physics of the Earth, vol. 22, no. 2, pp. 237-244, 1974.

[7] S. B. Sinha and K. A. Elsibai, "Reflection of thermoelastic waves at a solid half-space with two relaxation times," Journal of Thermal Stresses, vol. 19, no. 8, pp. 749-762, 1996.

[8] S. B. Sinha and K. A. Elsibai, "Reflection and refraction of thermoelastic waves at an interface of two semi-infinite media with two relaxation times," Journal of Thermal Stresses, vol. 20, no. 2, pp. 129-145, 1997.

[9] J. N. Sharma, V. Kumar, and D. Chand, "Reflection of generalized thermoelastic waves from the boundary of a half-space," Journal of Thermal Stresses, vol. 26, no. 10, pp. 925-942, 2003.

[10] M. I. A. Othman and Y. Song, "Reflection of plane waves from an elastic solid half-space under hydrostatic initial stress without energy dissipation," International Journal of Solids and Structures, vol. 44, no. 17, pp. 5651-5664, 2007.

[11] B. Singh, "Effect of hydrostatic initial stresses on waves in a thermoelastic solid half-space," Applied Mathematics and Computation, vol. 198, no. 2, pp. 494-505, 2008.

[12] B. Singh, "Reflection of plane waves at the free surface of a monoclinic thermoelastic solid half-space," European Journal of Mechanics, A/Solids, vol. 29, no. 5, pp. 911-916, 2010.

[13] M. E. Gurtin and W. O. Williams, "On the Clausius-Duhem inequality, Zeitschrift für angewandte Mathematik und Physik, vol. 17, no. 5, pp. 626-633, 1966.

[14] M. E. Gurtin and W. O. Williams, "An axiomatic foundation for continuum thermodynamics," Archive for Rational Mechanics and Analysis, vol. 26, no. 2, pp. 83-117, 1967.

[15] P. J. Chen and M. E. Gurtin, "On a theory of heat conduction involving two temperatures," Zeitschrift für angewandte Mathematik und Physik ZAMP, vol. 19, no. 4, pp. 614-627, 1968.

[16] P. J. Chen, M. E. Gurtin, and W. O. Williams, "A note on nonsimple heat conduction," Zeitschrift für angewandte Mathematik und Physik, vol. 19, no. 6, pp. 969-970, 1968.

[17] P. J. Chen, M. E. Gurtin, and W. O. Williams, "On the thermodynamics of non-simple elastic materials with two temperatures," Zeitschrift für angewandte Mathematik und Physik, vol. 20, no. 1, pp. 107-112, 1969. 
[18] W. E. Warren and P. J. Chen, "Wave propagation in the two temperature theory of thermoelasticity," Acta Mechanica, vol. 16, no. 1-2, pp. 21-33, 1973.

[19] B. A. Boley and I. S. Tolins, "Transient coupled thermoplastic boundary value problems in the half-space," Journal of Applied Mechanics, vol. 29, no. 4, pp. 637-646, 1962.

[20] P. Puri and P. M. Jordan, "On the propagation of harmonic plane waves under the two-temperature theory," International Journal of Engineering Science, vol. 44, pp. 1113-1126, 2006.

[21] R. Quintanilla and P. M. Jordan, "A note on the two temperature theory with dual-phase-lag delay: some exact solutions," Mechanics Research Communications, vol. 36, no. 7, pp. 796-803, 2009.

[22] H. M. Youssef, "Theory of two-temperature-generalized thermoelasticity," IMA Journal of Applied Mathematics, vol. 71, no. 3, pp. 383-390, 2006.

[23] R. Kumar and S. Mukhopadhyay, "Effects of thermal relaxation time on plane wave propagation under two-temperature thermoelasticity," International Journal of Engineering Science, vol. 48, no. 2, pp. 128-139, 2010.

[24] A. Magaña and R. Quintanilla, "Uniqueness and growth of solutions in two-temperature generalized thermoelastic theories," Mathematics and Mechanics of Solids, vol. 14, no. 7, pp. 622-634, 2009.

[25] H. M. Youssef, "Theory of two-temperature thermoelasticity without energy dissipation," Journal of Thermal Stresses, vol. 34, no. 2, pp. 138-146, 2011. 

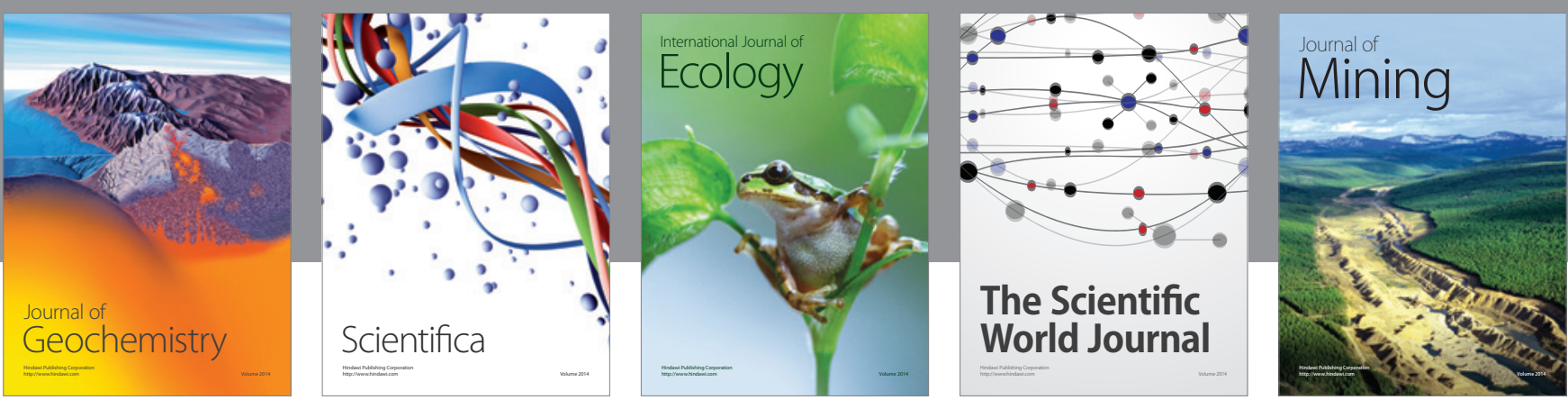

The Scientific World Journal
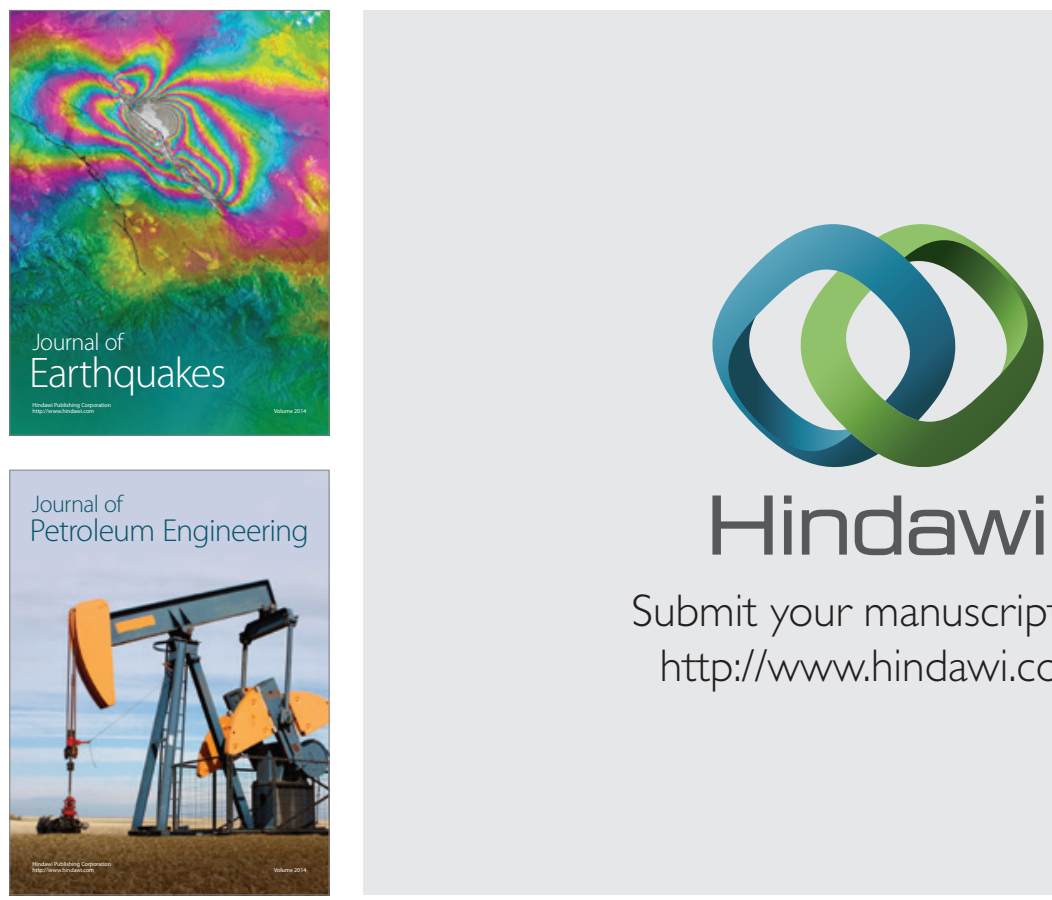

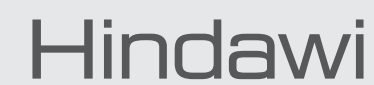

Submit your manuscripts at

http://www.hindawi.com
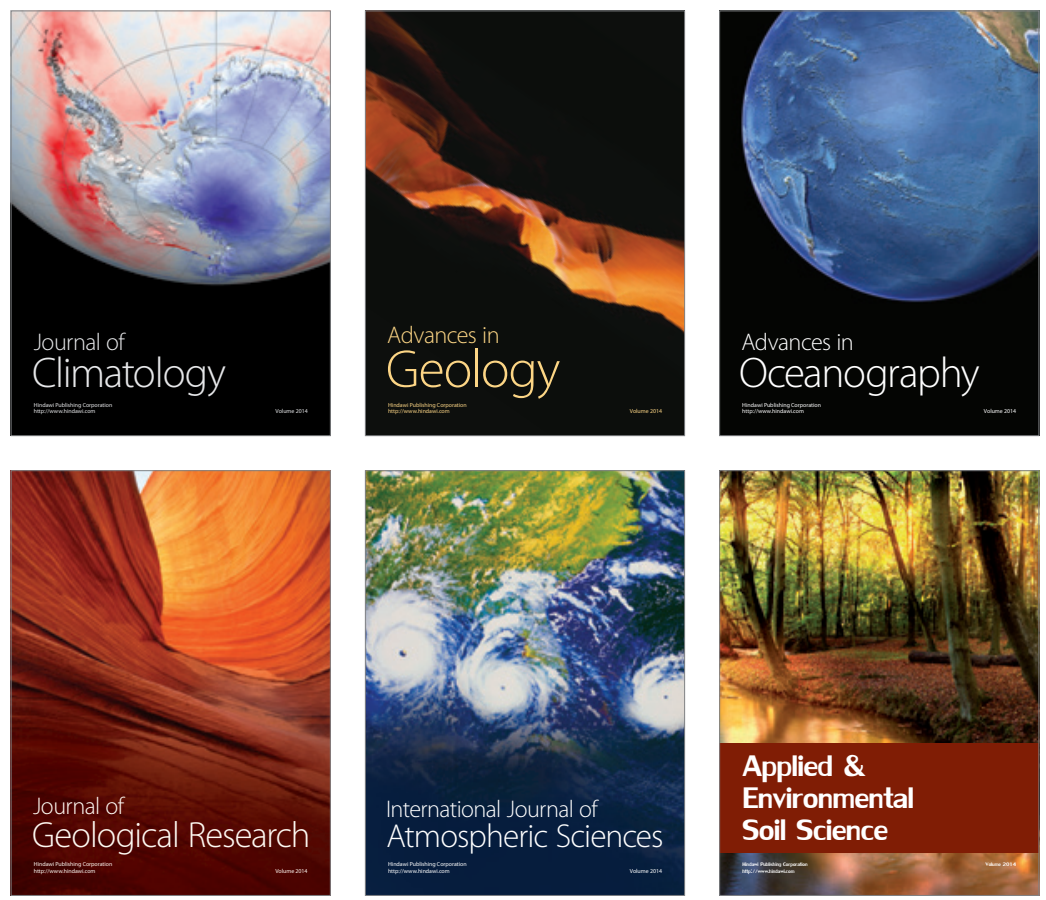
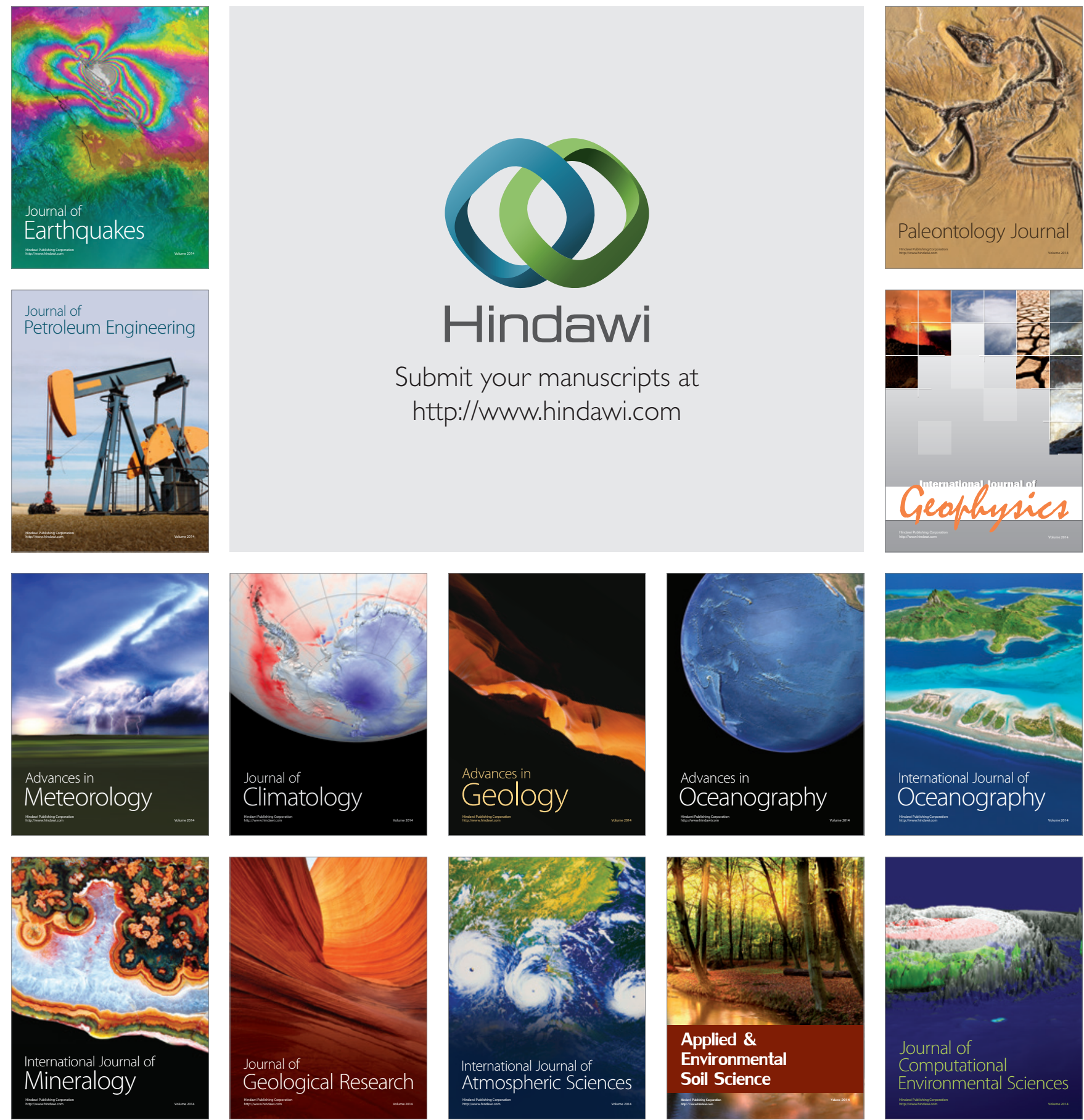